\title{
A Ten-Year Retrospective Study on the Clinical Features and Outcomes of Pediatric Intestinal Tuberculosis Admitted in UP-Philippine General Hospital
}

\author{
Eleonor G. Rodenas-Sabico, Germana Emerita V. Gregorio and Ma. Liza Antoinette M. Gonzales
}

Department of Pediatrics, College of Medicine and Philippine General Hospital, University of the Philippines Manila

\begin{abstract}
Objective. To describe the clinical, biochemical, microbiologic, radiologic and histological features and outcome of intestinal TB.

Methods. Medical records of patients diagnosed with intestinal TB were reviewed. Cases were considered bacteriologically-confirmed if intestinal tissue was positive on smear culture or polymerase chain reaction (PCR); and clinically-diagnosed if with clinical, histologic, and radiologic evidence of extra-pulmonary TB.

Results. Fifteen patients [Mean (SD) age: 13 (4) years; 53\% females] were included. One was bacteriologicallyconfirmed; and fourteen were clinically-diagnosed. Fever (87\%) and abdominal pain (73\%) were commonly seen. Seven (47\%) had anemia, 5 (33\%) leukocytosis and 10 (71\%) hypoalbuminemia. Eleven (73\%) were positive on smear or TB PCR of various specimens. Nine of 10 (90\%) patients with an abdominal CT scan had thickening of bowel loops. Three with intestinal biopsy demonstrated caseation granuloma. Fourteen patients were given quadruple anti-TB medications. Six had surgery, 8 had no disease-related complications, 4 died of sepsis and 3 were lost to follow up.
\end{abstract}

Conclusion. Intestinal TB presents with non-specific clinical and laboratory features. Radiologic findings may provide a clue to the diagnosis. Histologic confirmation in intestinal tissue was only seen in a few cases. The prognosis was favorable for patients who completed the anti-TB treatment.

Key Words: intestinal tuberculosis, extrapulmonary tuberculosis, Mycobacterium tuberculosis

\section{INTRODUCTION}

Oral presentation in the Fellows Forum, Philippine Society of Pediatric Gastroenterology, Hepatology \& Nutrition (PSPGHAN), August, 2018, Seda Hotel, Bonifacio Global City.

Research Poster Presentation at the Department of Pediatrics $33^{\text {rd }}$ Annual Research Week, September 2017, Philippine General Hospital; Philippine Society of Pediatric Gastroenterology, Hepatology \& Nutrition (PSPGHAN) $13^{\text {th }}$ Annual Convention, March 2018, Crowne Plaza, Manila; Pediatric Infectious Society of the Philippines, Inc. (PIDSP), $25^{\text {th }}$ Annual Convention, February 2018, Crowne Plaza, Manila.

Corresponding author: Germana Emerita V. Gregorio, MD

Department of Pediatrics

Philippine General Hospital

University of the Philippines Manila

Taft Avenue, Manila 1000, Philippines

Email: gvgregorio@up.edu.ph
Tuberculosis (TB) is a bacterial infection caused by Mycobacterium tuberculosis. It is a life-threatening disease with the lungs as the primary site, but it can spread and affect any organ of the body like the abdomen. Abdominal tuberculosis is seen in 1 to $3 \%^{1}$ of all TB cases. It refers to the involvement of the gastrointestinal tract and the solid organs, including the liver, spleen and pancreas, mesenteric glands, peritoneum and adjacent lymph nodes. On the other hand, intestinal tuberculosis is an infection that is limited to any part of the intestine, from the duodenum to the anal area with $85 \%$ of the cases occurring at the ileocecal area. ${ }^{2}$

Intestinal tuberculosis is uncommon and has been reported in only 18 to $20 \%$ of patients with abdominal tuberculosis. A study done among 5,911 Indian children with tuberculosis showed that 115 (1.9\%) had abdominal tuberculosis, of whom only $21(0.3 \%)$ had intestinal involvement. ${ }^{1}$ Intestinal tuberculosis among patients with 
abdominal tuberculosis was also reported in two of 10 Taiwanese and three of 13 children from Tunisia. ${ }^{3,4}$ In the registry of disease of 3,172,137 patients from 2007 January to 2016 December of the Philippine Pediatric Society, only $165(0.005 \%)$ were diagnosed with $\mathrm{TB}$ of the intestines, peritoneum and mesenteric glands (ICD A18.3). Children with intestinal tuberculosis may present either with nonspecific symptoms of fever, abdominal pain and weight loss or with abdominal obstruction or perforation requiring immediate surgical intervention. Hematologic findings are nonspecific and include leukocytosis, raised erythrocyte sedimentation rate, anemia and hypoalbuminemia..$^{1,5}$

The features of intestinal tuberculosis in children have not been described in literature and knowledge about the disease has primarily been from case reports or in case series of abdominal tuberculosis. ${ }^{6,7,8}$

In this retrospective study, the clinical, biochemical, microbiologic, radiologic, histological features and outcome of pediatric patients diagnosed with intestinal tuberculosis in our institution during the past 10 years were examined. Early recognition of symptoms and prompt intervention may improve the outcome of these patients.

\section{METHODS}

Subjects 0-18 years old referred with a final diagnosis of intestinal TB were identified based on the census of the Sections of Pediatric Gastroenterology, Hepatology and Nutrition and the Infectious and Tropical Diseases in Pediatrics. Patients may either be a case of isolated intestinal TB or disseminated tuberculosis with intestinal involvement. ${ }^{9}, 10$ The medical charts of these patients were retrieved and relevant information recorded in a patient datasheet. For all patients, pertinent clinical, biochemical, radiologic and microbiologic features and outcomes were obtained. This included exposure to any household member diagnosed with tuberculosis and presenting with intestinal symptoms such as anorexia, vomiting, abdominal pain, diarrhea or bowel incontinence as well as non-specific symptoms of fever, weight loss, decrease appetite or cough. Pertinent findings on physical examination were documented, including nutritional status and presence of abdominal distention, abdominal mass, ascites or enterocutaneous fistula (ECF). Radiologic results and other imaging studies such as abdominal contrast studies, ultrasound, and CT scans were noted. Reports of any histopathologic studies of the intestines or its surrounding tissues, obtained surgically or endoscopically, were recorded.

Patients were classified as either bacteriologicallyconfirmed if intestinal tissue sample was positive on AFB smear, culture or TB PCR; and clinicallydiagnosed if clinical, histologic and radiologic findings are consistent with active extra-pulmonary tuberculosis, and decision of the attending physician to treat with anti-tuberculosis drugs. These may consist of at least one of the following: (1) histologic evidence of caseation granuloma or Langhans multinucleated giant cell in the intestinal tissue or evidence of inflammation in any area of the intestine; (2) radiologic findings either on abdominal contrast studies, X-ray, ultrasound or CT scan demonstrating thickening, dilatation or palisading of bowel loops involving any part of the intestine; and (3) clinical improvement following anti-TB treatment including resolution of fever, decreased abdominal pain or improved appetite.

\section{Data analysis}

Data were described using means and standard deviations for continuous variables and frequencies (percentages) for categorical variables.

\section{RESULTS}

A total of fifteen (15) patients were diagnosed to have intestinal tuberculosis during the ten year period. Only one case was positive for acid-fast bacilli smear on intestinal tissue specimen but no TB culture was sent. Fourteen patients were classified as clinically-diagnosed: three (21\%) were based on histologic evidence and eleven (79\%) based on radiologic findings. Six (40\%) of the patients had isolated intestinal tuberculosis and nine (60\%) had disseminated tuberculosis with lung, heart, bone and genitourinary involvement (Table 1).

Clinical features. The mean (SD) age of the patients was $13( \pm 4.36)$ years, with the youngest at 1 year and the oldest 18 years old. Eight (53\%) were female. Of the fifteen (15), 6 (40\%) had a known history of TB exposure. The mean duration of symptoms before diagnosis was four $( \pm 6.03)$ months, from one month to 2 years from the onset of illness. Three of five patients had positive Mantoux test. The most common clinical presentation before admission was fever (87\%), abdominal pain (73\%) and abdominal distention (60\%). All of the patients had at least one of these three symptoms before the diagnosis. Only one case had fever with no abdominal pain and distension, but in the presence of other symptoms including decreased appetite, loose stools and pallor, which prompted further workup for intestinal tuberculosis.

In terms of physical examination, 6 (40\%) patients had severe malnutrition, 4 (27\%) had moderate acute malnutrition and 5 (33\%) had no wasting. The most common physical findings were abdominal tenderness (67\%), followed by abdominal distension (33\%) and abdominal mass (27\%). One case presented with an enterocutaneous fistula, abdominal pain and abdominal distension (patient \#5) after five months of fever.

Biochemical and Microbiologic features. The mean hemoglobin, white cell count and serum albumin are presented in Table 1. Seven (47\%) patients had anemia (hemoglobin of $<10 \mathrm{~g} / \mathrm{L}$ ), five (33\%) with leukocytosis 
Features and Outcome of Intestinal Tuberculosis in Children

Table 1. Summary of clinical, biochemical, radiologic, histologic and microbiologic features of 15 patients with intestinal tuberculosis

\begin{tabular}{|c|c|c|c|c|c|c|}
\hline Patient & Age (years) & Chief Complaints & Hemoglobin (g/L) & Leucocytes x $10^{9} / \mathrm{L}$ & Lymphocytes & Albumin (g/L) \\
\hline 1 & 13 & Vomiting & 129 & 8.70 & 0.08 & 34 \\
\hline 2 & 13 & Abdominal pain & 73 & 4.59 & 0.18 & 13 \\
\hline 3 & 12 & Weakness & 123 & 10.19 & 0.07 & 13 \\
\hline 4 & 12 & Abdominal distention & 99 & 10.30 & 0.06 & 14 \\
\hline 5 & 15 & Easy fatigability & 72 & 9.49 & 0.09 & 14 \\
\hline 6 & 17 & Abdominal distention & 105 & 7.74 & 0.07 & 17 \\
\hline 7 & 16 & Abdominal pain & 101 & 12.20 & 0.14 & 18 \\
\hline 8 & 14 & Abdominal pain & 113 & 3.80 & 0.34 & 27 \\
\hline 9 & 17 & Abdominal distention & 96 & 7.46 & 0.06 & 24 \\
\hline 10 & 11 & Abdominal pain & 127 & 6.00 & Result not available & 35 \\
\hline 11 & 13 & Abdominal pain & 84 & 4.57 & 0.05 & 28 \\
\hline 12 & 6 & Cough & 83 & 14.71 & 0.20 & Not done \\
\hline 13 & 18 & Abdominal pain & 123 & 12.08 & 0.09 & 33 \\
\hline 14 & 1 & Abdominal distention & 86 & 5.01 & 0.36 & 18 \\
\hline 15 & 17 & Abdominal distention & 133 & 3.35 & 0.26 & 34 \\
\hline
\end{tabular}

(white cell count of $>10 \times 10^{9} / \mathrm{L}$ ) and 10 of $14(71 \%)$ with hypoalbuminemia (serum albumin less than $30 \mathrm{~g} / \mathrm{L}$ ). Fourteen patients had lymphocyte value that were normal. Three patients had an erythrocyte sedimentation rate that was elevated. A total of eleven patients yielded positive results in acid-fast bacillus (AFB) smear and TB PCR (Table 2). In different specimens, the AFB smear was positive in 15 (33\%) of 46 determinations and the TB PCR in two $(29 \%)$ of seven samples. The latter was obtained from the enterocutaneous fistula and endotracheal tube. No specimen was sent for TB culture.

Imaging investigations. Of the 15 patients, one had normal chest X-ray result and 14 had findings suggestive of pulmonary tuberculosis. These included the presence of reticulonodular opacities in eight, pleural effusion in seven, pleural thickening in three and one case each with miliary seeds, cystic lucencies, fibrotic opacities, perihilar infiltrates and mediastinal mass.

All fifteen (15) patients had an abdominal ultrasound and CT scan. Among the 12 patients who underwent ultrasound imaging, four (33\%) had normal results. The most common abdominal ultrasound finding was ascites (42\%). On abdominal CT scan, 9 of 10 (90\%) patients had thickening of bowel loops in different areas of the colon
Table 2. Results of AFB smear and TB PCR of 11 patients diagnosed with intestinal tuberculosis

\section{Specimen}

AFB Smear, $n^{*}$ TB PCR, $n^{*}$

Pulmonary

\begin{tabular}{lcc} 
Sputum & $4 / 9$ & $0 / 1$ \\
Gastric & $3 / 5$ & - \\
Endotracheal tube & $0 / 1$ & $1 / 1$ \\
\hline Extrapulmonary & & \\
\hline Stool & $4 / 6$ & - \\
Urine & $1 / 13$ & - \\
Pericardial fluid & $0 / 1$ & $0 / 1$ \\
Peritoneal fluid & $0 / 3$ & $0 / 1$ \\
Pleural fluid & $0 / 1$ & $0 / 1$ \\
Intestinal tissue & $1 / 3$ & - \\
Enterocutaneous fistula drainage & $1 / 1$ & $1 / 1$ \\
Omentum & $1 / 1$ & - \\
Jackson-pratt drain fluid & $0 / 1$ & $0 / 1$ \\
Abdominal wound abscess/aspirate & $0 / 1$ & - \\
\hline Total, n (\%) & $15 / 46(33)$ & $2 / 7(29)$ \\
\hline
\end{tabular}

*number positive over the total number of specimen sent

including the site of the enterocutaneous fistula. Only one case had thickening of bowel loops (Figure 1) in the ileocecal area (patient \#4). Other CT scan findings included ascites (60\%) and omental thickening and nodularities (40\%). 


\begin{tabular}{|c|c|c|c|c|}
\hline Patient & Chest $\mathrm{X}$-ray result & Abdominal Ultrasound result & Abdominal CT scan result & $\begin{array}{l}\text { Microbiologic }(+) \text { result } \\
\text { suggestive of TB }(n=11)\end{array}$ \\
\hline 1 & Nodularities & Ascites & & \\
\hline 2 & Pleural thickening, nodularities & Normal & & Gastric AFB \\
\hline 3 & $\begin{array}{c}\text { Effusion, miliary seeds, } \\
\text { nodularities }\end{array}$ & & $\begin{array}{l}\text { Segmental thickening of sigmoid } \\
\text { and descending colon, ascites }\end{array}$ & ETA AFB and PCR \\
\hline 4 & Nodularities & & $\begin{array}{l}\text { Thickening of ileocecal portion, } \\
\text { ascites, lymphadenopathy }\end{array}$ & $\begin{array}{l}\text { Gastric, stool and } \\
\text { sputum AFB }\end{array}$ \\
\hline 5 & Normal & & $\begin{array}{l}\text { Diffuse bowel wall thickening, } \\
\text { enterocutaneous fistula, abscess }\end{array}$ & $\begin{array}{l}\text { Enterocutaneous } \\
\text { fistula AFB }\end{array}$ \\
\hline 6 & Effusion & Normal & Diffuse bowel wall thickening & Sputum AFB \\
\hline 7 & $\begin{array}{l}\text { Fibrotic opacities, } \\
\text { effusion, nodularities }\end{array}$ & Normal & & \\
\hline 8 & Nodularities & $\begin{array}{l}\text { Dilatation of bowel loops, ascites, } \\
\text { septations in abdominopelvic region }\end{array}$ & Omental thickening & $\begin{array}{l}\text { Stool, sputum, urine, } \\
\text { omentum AFB }\end{array}$ \\
\hline 9 & $\begin{array}{c}\text { Effusion, pleural thickening, } \\
\text { mediastinal mass }\end{array}$ & Thickened bowel serosa, ascites & $\begin{array}{l}\text { Mesenteric and omental } \\
\text { thickening and stranding }\end{array}$ & Stool AFB \\
\hline 10 & Effusion, nodularities & $\begin{array}{l}\text { Thickened bowel serosa, omental } \\
\text { thickening, palisading bowel loops }\end{array}$ & $\begin{array}{l}\text { Lymphadenopathy, ascites, } \\
\text { omental thickening }\end{array}$ & \\
\hline 11 & Effusion & Ascites and omental caking & $\begin{array}{l}\text { Diffuse thickening with focal } \\
\text { dilatation of the sigmoid colon, } \\
\text { nodularities of omentum, ascites }\end{array}$ & Gastric AFB \\
\hline 12 & $\begin{array}{c}\text { Cystic lucencies, nodularities, } \\
\text { pleural thickening }\end{array}$ & Abscess & $\begin{array}{l}\text { Small bowel loop wall thickening, } \\
\text { ascites, lymphadenopathy }\end{array}$ & \\
\hline 13 & Pulmonary tuberculosis & Normal & & Sputum AFB \\
\hline 14 & Perihilar infiltrates & $\begin{array}{l}\text { Dilatation of bowel loops, } \\
\text { air-fluid levels }\end{array}$ & & Intestinal tissue AFB \\
\hline 15 & Effusion & Ascites & $\begin{array}{l}\text { Segment thickening of the sigmoid and } \\
\text { descending colon, small bowel wall } \\
\text { thickening, ascites, omental thickening }\end{array}$ & Stool AFB \\
\hline
\end{tabular}

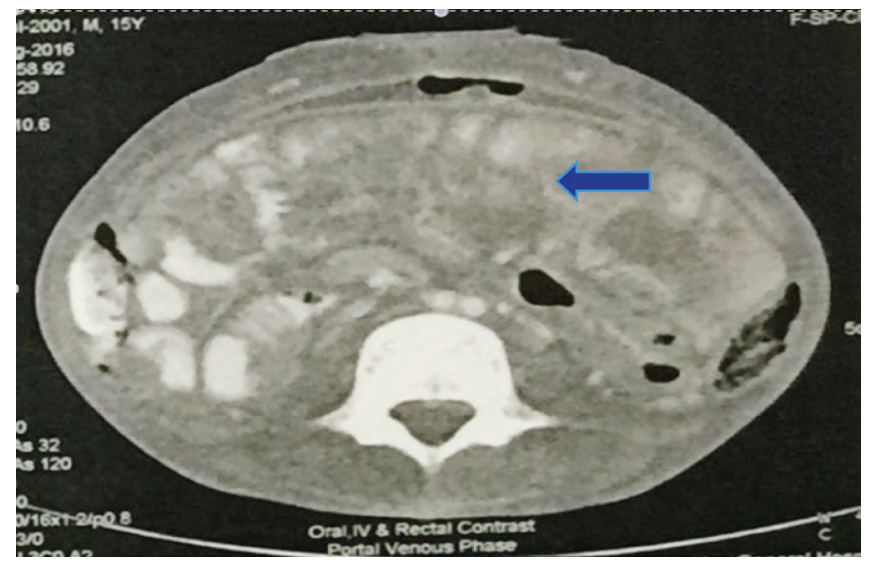

Figure 1. Diffuse bowel wall thickening (arrow) noted on Abdominal CT scan.

One patient (patient \#1) underwent intestinal series as the abdominal X-ray showed distended bowel loops. The result showed wall thickening at the proximal two-thirds of the ascending colon, including the cecum and distal ileum. Another (patient \#3) had an upper gastrointestinal series done due to signs of gastric outlet obstruction with a note of a filling defect at the duodenal area.
Histologic findings. Three subjects had an intestinal biopsy taken; two obtained surgically during exploratory laparotomy at the ileocecal area and ascending colon and one taken endoscopically at the second portion of the duodenum. All showed presence of caseation granuloma with Langans type multinucleated giant cells and necrosis (patients \#3,7,13).

Treatment. Of the 15 patients, 14 were treated with quadruple anti-tuberculosis treatment (Isoniazid, Rifampicin, Ethambutol and Pyrazinamide), one died from generalized peritonitis even before the initiation of treatment. Two of the 14 patients developed adverse drug reactions, i.e., jaundice and rash, but which improved with rechallenge using individual drugs.

Six patients underwent surgery and two underwent the endoscopic procedure. Surgery included bowel resection on two cases secondary to matted terminal ileum and cecum and the presence of a mass obstructing the ileocecal area. Another two had peritoneal lavage (Figure 2), one had adhesiolysis, and one had an incision and drainage of intra-abdominal abscess at the right lower quadrant. The endoscopic procedure included a colonoscopy for a rectovaginal fistula in a 13-year old patient (patient \#2) who developed fecaloid material at the vaginal area during confinement. Colonoscopy showed a fistula $8 \mathrm{~cm}$ from the anal verge with multiple $0.2-0.3 \mathrm{~mm}$ 


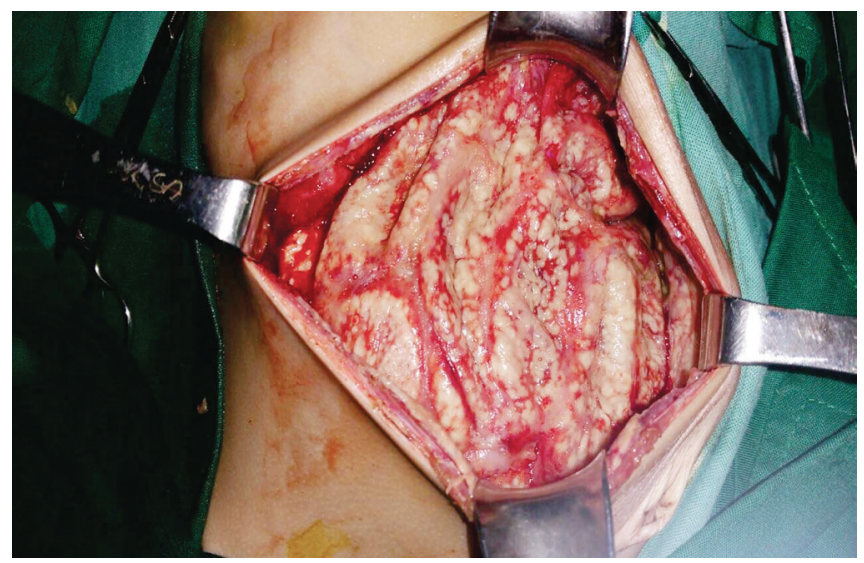

Figure 2. Intestinal perforations with abscesses on exploratory laparotomy (patient \#14).

polyps at the rectum and ileum, which were considered as inflammatory pseudopolyps. A 12-year-old female patient (patient \#3) underwent esophagogastroduodenoscopy (EGD) after an upper gastrointestinal series that showed a filling defect at the duodenal area. This patient had a smooth circumferential mass with nodularities from the pylorus to the second part of the duodenum.

Outcome. Of the 15 patients, 11 were discharged, four died due to sepsis. Only eight of the 11 patients followed up with a mean duration of $6.25( \pm 6.28)$ months with no complications. Three (3) were lost to follow up.

\section{DISCUSSION}

Our study showed intestinal tuberculosis as a rare disease in the pediatric age group in PGH, the largest tertiary government medical center in the Philippines. The 15 patients comprised $5 \%$ of the 300 tuberculosis cases admitted at the pediatric ward during the same ten-year period.

There were limited reports of intestinal tuberculosis in children and most were described as part of abdominal tuberculosis, including nine (9) of $153^{1,11}$ patients from India, three (3) of $13^{4}$ from Tunisia, two (2) of $10^{3}$ in Taiwan and two (2) of $6^{12}$ in Canada. A recent study from India ${ }^{2}$ also reported five (5) children with primary duodenal tuberculosis.

The mean age of presentation upon admission in our study was 13 years, which was comparable with the two cases of intestinal tuberculosis from Taiwan, with the ages of 12 and 17 years old, but older than the mean age of 5.2 years in five (5) patients with duodenal tuberculosis. ${ }^{3}$ The initial signs and symptoms are usually non-specific, which led to a delay in diagnosis. Of the seven intestinal tuberculosis cases in Tunisia, Taiwan and Canada, three presented with abdominal pain, two with asthenia and weight loss and one patient each with an acute abdomen and abdominal mass. All five patients in India with duodenal tuberculosis had gastric outlet obstruction with vomiting and abdominal pain at the onset of illness. The mean duration was 85 days before diagnosis. In our case series, the majority presented with a lengthy history of fever, abdominal pain and abdominal distension, from one month to two years. The protracted course of the illness should therefore alert physicians to perform further workups although it is of note that some cases may mimic a surgical abdomen ${ }^{3,12}$ as was observed in one patient in our study (patient \#3).

The definitive diagnosis of intestinal tuberculosis is confirmed by demonstration of Mycobacterium tuberculosis in the intestinal tissue; and presumptively in our setting, by the presence of caseation granuloma on histology as seen in three of our cases (patients \#3,7,13). However, only three cases had intestinal tissue specimens sent for microbiologic and histologic studies and TB PCR was not done on the specimens submitted. It is also limited by the sensitivity of AFB smear, which is 32 to $35 \%$, and PCR tests approximately $65 \% .{ }^{12}$ In our series, around $30 \%$ of our patients had positive AFB smear and PCR tests on various specimens submitted, such as enterocutaneous fistula drainage, omentum and abdominal wound abscess/aspirate (Table 2). As in previous studies, our study showed that $50 \%$ of our patients had anemia, and the majority had hypoalbuminemia, which attributed to the nutritional deficiency and chronicity of the illness. Yet, despite the presence of tuberculous infection in which lymphocytosis was expected, only a third of our patients had leukocytosis and all had normal lymphocyte count.

Once abdominal tuberculosis is suspected, imaging studies in the form of an ultrasound or CT scan should be performed. The results are usually non-specific, although these may give a clue to the diagnosis. Ascites was the most common finding in both the abdominal ultrasound and CT scan in our patients, followed by the presence of thickening of the bowel loops and omental thickening and caking as a result of inflammation. Ascites, brought about by hypoalbuminemia, were reported in three cases of intestinal tuberculosis in Tunisia and bowel wall thickening in another two patients in Canada. The ileocecal region which is the most common site of intestinal tuberculosis, was the area of involvement in two of our patients, as seen both on imaging and during laparotomy. Predilection of the bacillus for the ileo-cecum was secondary to the abundance of lymphoid tissue, the high rate of absorption in the region and the minimal digestive activity causing increased contact time between the bacteria and intestinal mucosa. In other cases reported in literature, ${ }^{3,11}$ specific imaging findings of those with intestinal tuberculosis could not be ascertained as the study collectively reported the results of patients with abdominal tuberculosis.

Management of intestinal tuberculosis is mainly medical. Endoscopy or surgical intervention is only performed when warranted. Treatment consists of quadruple drugs consisting of six months of Isoniazid and Rifampicin and 2 months of Pyrazinamide and Ethambutol as what we have done in 14 of our patients. The medication was well tolerated except for two (14\%) who developed jaundice and rash but improved after the dose of the medications were reduced. 
Hepatotoxic complications such as jaundice and elevation of transaminases have been observed in $1.84 \%{ }^{13}$ of the patients on anti-tuberculosis drug, usually Isoniazid. ${ }^{13,14}$ Druginduced rash is associated with any of the four drugs and has been reported in $1 \%{ }^{13}$ of cases. Some authors recommend the addition of corticosteroids in patients with peritoneal disease to reduce subsequent complications of adhesions, although no clinical studies have been performed to show the benefit. ${ }^{15}$

In our patients, the upper and lower gastrointestinal endoscopies were performed as a diagnostic procedure. This allowed us to do a biopsy of a duodenal mass that we suspected based on the radiologic images. The colonoscopy determined the extent of a rectovaginal fistula and histologic findings revealed the presence of inflammatory polyps and ileocolitis with no granuloma. Intestinal tuberculosis presenting with spontaneous rectovaginal fistula has been previously reported in a seven-year-old child in Nigeria. ${ }^{7}$

Surgery is usually reserved for patients developing complications such as obstruction, perforation or uncontrolled hemorrhage or in those in whom the diagnosis could not be ascertained by any means. Two of our patients required surgical resection for obstructing lesions, although some authors have advocated conservative treatment even for intestinal obstruction as lesions have been shown to improve with anti-tuberculosis treatment. ${ }^{16,17}$ Of 34 patients with symptoms of intestinal obstruction and radiologic evidence of intestinal stricture secondary to tuberculosis, 31 (91\%) showed clinical improvement after one year of treatment and three required resection of the involved segment.

The prognosis is favorable for patients with intestinal TB who complete the course of treatment. Eight of our 11 patients who were discharged and finished the treatment are presently well and have not developed any further problems related to the disease. A similar experience was reported in 22 adult patients in Hong Kong who had resolution of abdominal symptoms after anti-tuberculosis treatment. There was no mortality related to $\mathrm{TB}$, although two died from colon cancer before the completion of the treatment.

In conclusion, intestinal tuberculosis presents with nonspecific clinical and laboratory features and a high index of suspicion is needed to make a diagnosis, especially for children who present with prolonged fever, abdominal pain and abdominal distension. Specific radiologic findings may provide a clue to the diagnosis. The presence of caseation granuloma on histology gives presumptive evidence of the disease but a definitive diagnosis is only made by the presence of Mycobacterium tuberculosis in the intestinal tissue. The prognosis is favorable for patients who complete the course of treatment while surgery is reserved for those who develop disease-related complications.

The study is limited by its retrospective nature and the small sample size. No statistical analysis could be done to determine the association of the different features and outcome of the patient. A multicenter prospective study should be made in the future.

\section{Statement of Authorship}

All authors participated in data collection and analysis and approved the final version submitted.

\section{Author Disclosure}

All authors declared no conflicts of interest.

\section{Funding Source}

This paper has been granted partial research funds by the Pediatric Infectious Disease Society of the Philippines, Inc. (PIDSP).

\section{REFERENCES}

1. Basu S, Ganguly S, Chandra PK, Basu S. Clinical profile and outcome of abdominal tuberculosis in Indian children. Singapore Med J. 2007; 48(10):900-5.

2. Upadhyaya VD, Kumar B, Lal R, Sharma MS, Singh M, Rudramani. Primary duodenal tuberculosis presenting as gastric-outlet obstruction: Its diagnosis. Afr J Paediatr Surg. 2013; 10(2):83-6.

3. Lin YS, Huang YC, Lin TY. Abdominal tuberculosis in children: a diagnostic challenge. J Microbiol Immunol Infect. 2010; 43(3):188-93. doi: 10.1016/S1684-1182(10)60030-8.

4. Tinsa F, Essaddam L, Fitouri Z, Brini I, Douira W, Ben Becher S, et al. Abdominal tuberculosis in children. J Pediatr Gastroenterol Nutr. 2010; 50(6):634-8. doi: 10.1097/MPG.0b013e3181b6a57b..

5. Debi U, Ravisankar V, Prasad KK, Sinha SK, Sharma AK. Abdominal tuberculosis of the gastrointestinal tract: Revisited. World J Gastroenterol. 2014; 20(40):14831-40.

6. Mahajan D, Nigam S, Kohli K. Abdominal tuberculosis presenting as ileocolic intussusception in an infant. Pediatr Dev Pathol. 2007; 10(6):477-80. doi:10.2350/06-06-0107.1.

7. Usang UE, Inyang AW, Nnoli MA, Emehute, JDC, Nwachukwu IE. Abdominal tuberculosis presenting with spontaneous recto-vaginal fistula in a 7 year old child- A case report. Med Res Chron. 2016; 3(5):323-7.

8. Boukthir S, Mrad SM, Becher SB, Khaldi F, Barsaoui S. Abdominal tuberculosis in children. Report of 10 cases. Acta Gastroenterol Belg. 2004; 67(3):245-9.

9. Wang YA, Yu WY. Isolated colonic tuberculosis. Postgrad Med J. 1987 May; 63(739):403-4. doi: 10.1136/pgmj.63.739.403.

10. Wang JY, Hseuh PR, Wang SK, Jan IS, Lee LN, Liaw YS, et al. Dissseminated Tuberculosis. A 10-year Experience in a Medical Center. Medicine (Baltimore). 2007; 86(1):39-46.

11. Malik R, Srivastava A, Yachha SK, Poddar U, Lal R. Childhood abdominal tuberculosis: Disease patterns, diagnosis and drug resistance. Indian J Gastroenterol. 2015; 34(6):418-25.

12. Delisle M, Sequin J, Zeilinski D, Moore DL. Pediatric abdominal tuberculosis in developed countries: case series and literature review. Arch Dis Child. 2016; 101(3):253-8.

13. Li Y, Zhu Y, Zhong Q, Zhang X, Shu M, Wan C. Serious Adverse Reactions from Anti-Tuberculosis Drugs Among 599 Children Hospitalized for Tuberculosis. Pediatric Infect Dis J. 2017; 36(8):7205. doi: 10.1097/INF.0000000000001532.

14. Shakya R, Rao BS, Shrestha B. Incidence of hepatotoxicity due to antitubercular medicines and assessment of risk factors. Ann Pharmacother. 2004; 38(6):1074-9.

15. Sood R. Diagnosis of Abdominal Tuberculosis: Role of Imaging. Journal Indian Academy of Clinical Medicine. 2001; 2(3):169-177.

16. Anand BS, Nanda R, Sachdev GK. Response of tuberculous stricture to antituberculous treatment. Gut. 1988; 29(1):62-69. doi: 10.1136/ gut.29.1.62

17. Leung VK, Law ST, Lam CW, Luk IS, Chau TN, Loke TK, et al. Intestinal tuberculosis in a regional hospital in Hong Kong: a 10-year experience. Hong Kong Med J. 2006; 12(4):264-71. 